\title{
Naturheilkundliche Behandlung der Erschöpfung
}

\author{
Ein Fall aus der Praxis: Multimodales Vorgehen mit Mikronährstoffsubstitution, \\ Ernährungsumstellung, Coaching, Achtsamkeitstraining, Wiederaufnahme des \\ Ausdauertrainings sowie Akupunkturbehandlung führt zur raschen Beschwerde- \\ besserung
}

Autorin: Ruth Biallowons

\section{ZUSAMMENFASSUNG}

Erschöpfte Patienten sind mit einem ganzheitlichen Therapieansatz in der Regel gut zu behandeln, wie die Erfahrung aus unserer Praxis zeigt. Eine Erschöpfung ist immer subjektiv, objektive Messmethoden existieren bisher nicht. Der erste Schritt ist, die Beschwerden des Patienten ernst zu nehmen. In unserer Praxis hat sich ein multimodales Vorgehen bewährt: Einer ausführlichen Anamnese u. a. mit Psychoedukation folgt eine erweiterte Diagnostik. Die multimodale Therapie umfasst die Ernährungsberatung, Stressmanagement, ggf. Vermittlung eines Coachings, Achtsamkeitsschulung, Bewegung, ggf. Mikronährstoffsubstitution, Akupunktur zum vegetativen Ausgleich. Ein aktives Mitwirken des Patienten ist wesentlich für den Therapieerfolg und stärkt die Selbstwirksamkeit.

\section{ABSTRACT}

Normally, exhausted patients can be treated well with a holistic therapeutic approach, as experience from our practice shows. An exhaustion is always subjective. So far, objective measuring methods do not exist. The first step is to take the discomforts of the patient seriously. In our practice, a multimodal procedure has proven to be effective: A detailed anamnesis e.g. with psychoeducation is followed by an enhanced diagnosis. The multimodal therapy includes nutrition counseling, stress management, provision of a coaching, where appropriate, mindfulness training, exercise, micronutrient substitution, where appropriate, acupuncture for vegetative balance. An active contribution of the patient is essential for the therapeutic success and strengthens self-efficacy.

Keywords

Exhaustion, naturopathy, orthomolecular medicine, multimodal therapy.

Erschöpfung, Naturheilkunde, Orthomolekulare Medizin, multimodale Therapie.

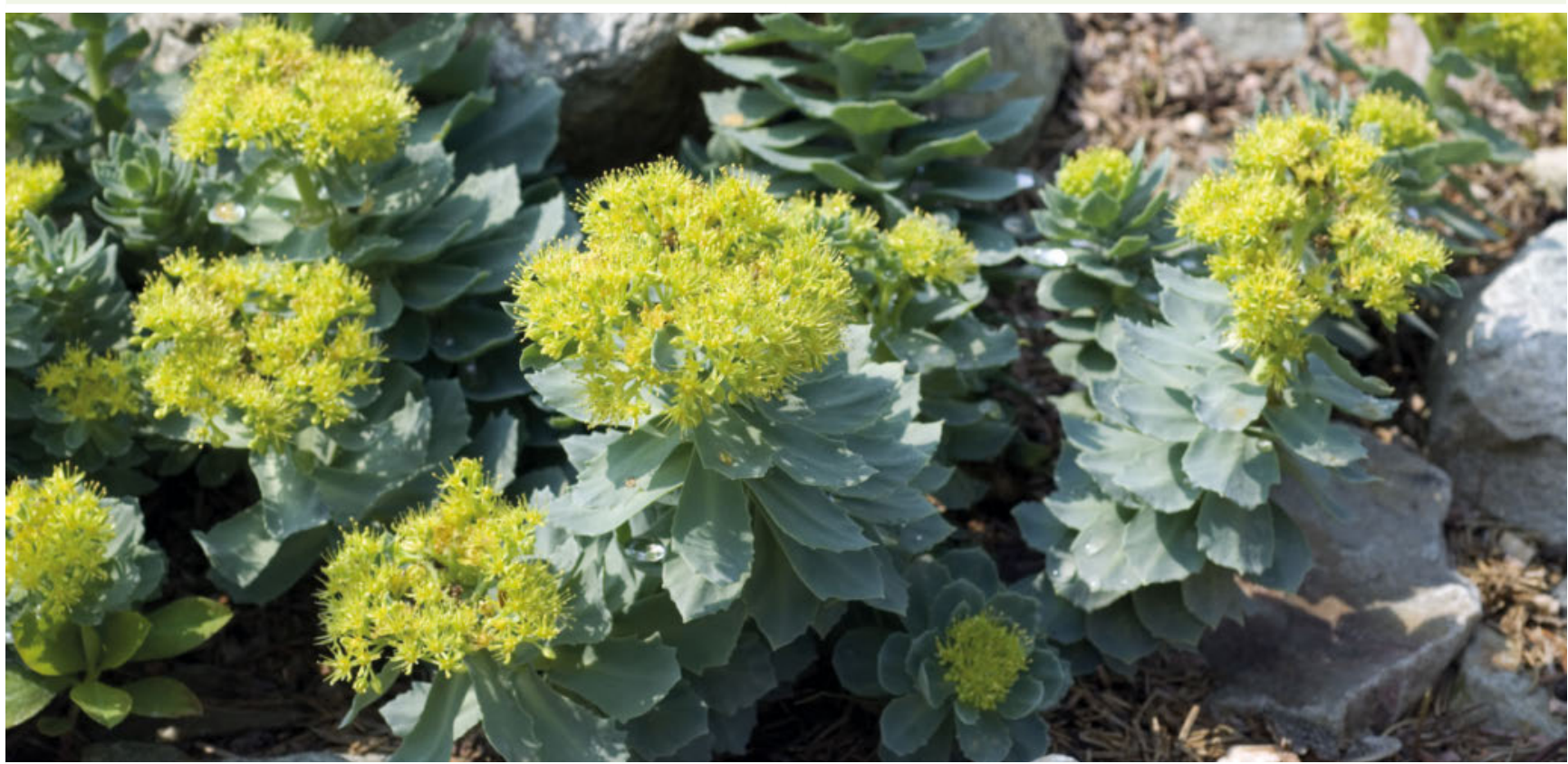

- Abb. 1 Rhodiola rosea (Rosenwurz) kann ergänzend bei stressbedingter Erschöpfung eingesetzt werden. @ emer/Adobe Stock

Erschöpfung und verwandte Beschwerden sind ein zunehmendes Phänomen. Die Grenzen zwischen Erschöpfung, Burnout, depressiven Verstimmungen bis hin zu einer manifesten Depression sind fließend und oftmals schwer einzuschätzen. Im weltweiten Vergleich hält Deutschland die meisten Krankenhausbetten für psychosomatische Er- 
krankungen vor [1]. Das wirft die Frage auf, warum wir in Deutschland so massiv von psychosomatischen Erkrankungen betroffen sind.

Einen steigenden Anteil haben sicherlich gesellschaftliche Entwicklungen unserer Zeit wie Digitalisierung, Multitasking und die Schnelligkeit, die unser tägliches Leben immer mehr prägen. Und sie stehen im Gegensatz zu unseren deutschen Tugenden, die wir in die Wiege gelegt bekommen: sei gefällig, pünktlich, perfekt, akkurat, stets höflich und tanze bloß nicht aus der Reihe. Diese Ansprüche aufrechtzuerhalten wird für viele Menschen immer schwieriger, sie haben das Gefühl, den Anforderungen nicht mehr gewachsen zu sein und fühlen sich überfordert, obwohl sie meist Großartiges leisten und auf vielen Hochzeiten gleichzeitig tanzen. Die Wahrheit ist jedoch, dass unser Gehirn kein Multitasking beherrscht, auch das weibliche nicht.

Wie gut wir in diesem System funktionieren, hängt von der individuellen Wahrnehmung der eigenen Situation und des eigenen Befindens ab. Außerdem spielt die ebenfalls individuell unterschiedlich ausgeprägte Resilienz eine Rolle. Wie stark sie ausgeprägt ist hängt davon ab, in welchem Maße wir gelernt haben, mit Stressoren, Diskrepanzen und Konflikten umzugehen. Wer hat nicht schon Sätze gehört wie „reiß dich zusammen“, „hör auf zu heulen“ oder „das war nicht schlimm“ - klassische Anweisungen, die eigenen Emotionen als solche nicht ernst zu nehmen bzw. sie zu unterdrücken.

Die Erkenntnis, dass unterdrückte Gefühle krankmachen können, ist nicht neu, ebenso wenig, dass sie sich massiv schädigend auf körperlicher und seelischer Ebene auswirken können. Ein Symptom kann eine Erschöpfung sein. Sie schreitet meist schleichend voran. Initial sind die Beschwerden gut zu kompensieren. Hält sie über längere Zeit an, können sich Symptome wie Schmerzen, Schlafstörungen oder auch Aggressionen meist ebenso schleichend zuspitzen und beispielsweise zu Burnout, Depression oder chronischen Schmerzzuständen führen.

Viele Patienten berichten, dass sie bereits Ärzte wegen einer Erschöpfung aufgesucht haben und mit dem Rat „Sie brauchen mal Urlaub“, oder „nehmen Sie sich eine Auszeit“ aus der Sprechstunde gegangen sind. Andere erzählen, dass sie ein Antidepressivum verschrieben bekamen oder eine Überweisung zum Psychiater erhielten und letztendlich genauso hilflos wie vorher dastanden.

Ein unterschätztes Problem bei diesen Herangehensweisen ist das Nichternstnehmen der Beschwerden. Die Hilfesuchenden fühlen sich nicht wertgeschätzt, allein gelassen und letztlich noch unzureichender als vorher. Dabei ist die Behandlung einer solchen Erschöpfung mit einer ganzheitlichen Strategie meist weniger kompliziert, wenn ver- sucht wird herauszufinden, welche Faktoren zur Erschöpfung geführt haben.

\section{Therapiestrategien in der naturheilkundlichen Praxis}

In unserer Praxis hat sich ein multimodales Vorgehen bei Patienten mit Erschöpfung bewährt.

Zunächst ist es wichtig, die Patienten mit ihren individuellen Beschwerden ernst zu nehmen. Die Erschöpfung ist grundsätzlich subjektiv, ebenso wie Schmerzen subjektiv sind. Bislang existieren keine objektivierbaren Messmethoden. In einer ausführlichen Anamnese, für die wir uns ca. 1 Stunde Zeit nehmen, führen wir auch eine ausführliche Psychoedukation durch. Die Anamnese gibt meist schon Hinweise auf mögliche Stressoren, Copingstrategien (wenn vorhanden) oder generelle Mängel, Ernährungsdefizite, Bewegungsmangel, berufliche, familiäre und emotionale Belastungen. Die Psychoedukation kann mögliche Vorurteile und auch Selbstvorwürfe, die sich die Patienten häufig aufgrund der bestehenden gesellschaftlichen Reaktionen und Haltungen machen, entkräften. In der Psychoedukation geht es darum, den Patienten darüber aufzuklären, wie die Beschwerden entstehen konnten und welche Zusammenhänge bestehen. Das Wissen um diese Zusammenhänge kann den Patienten unterstützen, seine Erschöpfung anzuerkennen und die Basis dafür schaffen, Hilfe anzunehmen. Die Therapie kann nur erfolgreich und nachhaltig sein, wenn die Patienten selbst aktiv mitwirken. Zudem wird die Selbstwirksamkeit gestärkt.

Neben den üblichen schulmedizinischen Check-ups und Laboruntersuchungen führen wir zusätzliche naturheilkundliche Diagnostik durch.

\section{Naturheilkundliche Diagnostik}

Herzfrequenzvariabilitätsmessung (HRV)

Mithilfe der HRV kann die Regulationsfähigkeit des vegetativen Nervensystems gemessen und Dysregulationen aufgedeckt werden. Bis es zu einer Erschöpfung kommt, lief der Körper wochen-, monate- oder sogar jahrelang auf Hochtouren. Das vegetative Nervensystem wird dadurch in seiner Regulationsfähigkeit geschwächt, eine Entspannung durch den Parasympathikus tritt häufig nicht mehr ein. Patienten berichten von Schlafstörungen, Herzrasen, Unruhezuständen, Angespanntheit und mangelnder Regenerationsfähigkeit.

\section{Erweiterte Mikronährstoffanalyse}

Ist der Organismus überwiegend durch den Sympathikus gesteuert, laufen alle Stoffwechselprozesse auf Hochtouren. Das bedeutet, dass ein erhöhter Bedarf an bestimmten Vitaminen und Mikronährstoffen besteht. Die zusätzlich benötigte Menge an Mikronährstoffen ist über die Ernährung oft nicht zu decken, auch weil Menschen in stressigen 
Situationen dazu neigen, nicht ausgewogen zu essen oder die Anspannung am Abend mit alkoholischen Getränken zu lösen versuchen. Das wiederum kann den Schlaf beeinträchtigen, der gerade in Stresssituationen für die Regeneration des Organismus wichtig ist.

Wir führen eine Vollblutmineralanalyse durch, die die intrazellulären Mineralstoffe Natrium, Kalium, Kalzium, Magnesium, Kupfer, Zink und Selen enthält. Zusätzlich dazu bestimmen wir folgende Parameter im Serum: Vitamin D, B-Vitamine, Coenzym Q10, Carnitin, belastende Schwermetalle, oxidiertes LDL, Homa-Index, Lipoprotein A, Fettsäureprofil mit Omega-3-Index und Omega6/Omega3Quotient, Morgen-Cortisol, ACTH, TSH, fT3, fT4, sowie Schilddrüsenantikörper, Zonulin im Serum, Histamin im Serum.

Häufig zeigen sich bei erschöpften Patienten Mängel oder Normwerte im unteren Bereich, die keinesfalls ausreichen, um in Belastungssituationen den Körper ausreichend zu versorgen.

\section{Neurostressprofil}

Im Neurostressprofil wird ein Cortisol-Tagesprofil aus dem Speichel (3 Messungen zu den Zeitpunkten 8h, 13h, 20h) bestimmt, das einen Anhalt für die Cortisolausschüttung über den Tag und die notwendige Absenkung zur Nacht gibt. Zusätzlich wird über den 2. Morgenurin die Menge von Serotonin, Adrenalin, Noradrenalin und Dopamin bestimmt sowie die Verhältnisse von Adrenalin/Noradrenalin und Serotonin/Dopamin. Häufig zeigt sich hier bereits die Erschöpfung der Neurotransmitter in Form eines Serotoninmangels sowie der Katecholamine über Adrenalinmangel an. Diese Ergebnisse geben weitere Hinweise auf eine Erschöpfung. Hat man den Verdacht einer Nebennierenrindeninsuffizienz ist die Bestimmung des 24h-Cortisol im Urin ergänzend notwendig.

\section{Mikrobiomanalyse}

In Stresssituationen kann die Mikrobiota ins Ungleichgewicht geraten, Nährstoffe können nicht ausreichend vom Organismus aufgenommen werden.

Liegen bei den Patienten Mikronährstoffmängel vor, die über die Ernährungsgewohnheiten oder trotz bestehender Mikronährstoffsubstitution nicht erklärbar sind, kann eine Mikrobiomanalyse sinnvoll sein, um eine enterale Dysbiose oder ein Leaky-gut-Syndrom auszuschließen. Auch bei neu auftretenden Allergien, Infektanfälligkeit oder Verdauungsstörungen ist eine Mikrobiomanalyse empfehlenswert.

\section{Therapieansätze}

Die Therapie erfolgt multimodal. Neben der Behandlung der körperlichen Beschwerden und der Substitution von Mikronährstoffen, liegt ein Schwerpunkt auf der Beratung zu Lebensstilfaktoren: Ernährungsberatung, Anleitung zur
Entspannung, Weiterleitung an kooperierende Coaches, Einbindung in unseren praxisinternen Achtsamkeitskurs sowie die umfassende Aufklärung über die Entstehung und den Umgang mit den vorhandenen Beschwerden bis hin zur Sportlerberatung. Zum vegetativen Ausgleich und zur Schmerzreduktion setzen wir häufig Akupunktur ein. In regelmäßigen, engmaschigen Kontrollterminen in der Anfangsphase können wir den Patienten individuell und professionell begleiten.

\section{Ein Fall aus der Praxis}

\section{Anamnese}

Herr A.J. ist ein Mittdreißiger, steht mit beiden Beinen im Job und trägt Personalverantwortung. Seine Frau hat gerade das 2 . Kind zur Welt gebracht und die Familie hat eine Eigentumswohnung gekauft. Bis zu diesem Zeitpunkt war Herr J. nie krank. Er kommt zum ersten Gespräch in unserer Praxis in Begleitung seiner Ehefrau. Sie bestätigt, dass es ihm nicht gut gehe. Sein Hausarzt habe ihm geraten, in Urlaub zu fahren, es sei ja schließlich sonst alles in Ordnung.

Er berichtet, sich zunehmend erschöpft, müde, ausgelaugt und lustlos zu fühlen. Im Job bringt er volle Leistung, sei aber nicht mehr so gut in seinen Führungsqualitäten. Wegen der andauernden Erschöpfung könne er seinen eigenen Ansprüchen nicht mehr richtig genügen. Die Aufgaben wachsen ihm allmählich über den Kopf. Aufgrund dieser Belastungen habe er Schlafstörungen, er liege nachts stundenlang grübelnd wach und erscheint eindeutig übernächtigt. Morgens ist er wie gerädert, missmutig gelaunt und sei für die Familie schlecht zu ertragen. Die Ehefrau berichtet, dass er sonst ein toller, ausgeglichener Mann und Vater sei, jetzt aber oft passiv aggressiv reagiere. Schöne Momente seien immer seltener. Ein Urlaub hätte keine Besserung gebracht.

Herr J. leidet zunehmend unter Kopf- und vor allem Schulter- und Nackenschmerzen. Er verspüre permanent eine innere Unruhe und sei einfach nicht mehr er selbst. Im Job war er immer sehr ehrgeizig, strebte nach Perfektion, vermied Konfliktsituationen. Er berichtet, früher regelmäßig gejoggt zu sein, aufgrund des stressigen Jobs und der Kinder sei dies aber immer weniger geworden und er habe schließlich ganz aufgehört.

Nun stand der nächste Karriereschritt an, den er sich selbst nicht mehr zutraute, was ihn sehr verunsicherte, da er ja bisher immer alle Herausforderungen gut gemeistert habe. Er sucht unsere Praxis auf, weil er sich eine andere Sichtweise auf das Wesen seiner Beschwerden erhofft.

In einem ersten ausführlichen Gespräch erläutere ich dem Patienten die Zusammenhänge von Stress, körperlichen Beschwerden, Emotionen und inneren Stressoren/Antreibern. Durch das Gespräch konnte ich Herrn J. die Angst vor 
einer chronischen andauernden Erkrankung nehmen. Wir räumten der Erschöpfung eine Berechtigung ein, da diese ja nicht ohne Grund entsteht.

\section{Diagnostik}

In einem umfassenden Check-up konnten organische Ursachen für die Erschöpfung ausgeschlossen werden.

\section{Labor}

In unserer Praxis untersuchen wir dazu neben großem Blutbild, Leber-, Nieren- und Pankreas-Werten, Eiweißelektrophorese sowie Cholesterinwerten und Triglyzeriden grundsätzlich die Schilddrüsenmarker (TSH, fT3, fT4, Schilddrüsenantikörper) zum Ausschluss eines nicht entdeckten Autoimmungeschehens, Vitamin D, Homocystein, Vitamin $B_{12}, B_{6}, B_{1}$ und Folsäure, bei Depression/Erschöpfung auch Vitamin $B_{3}$. Dazu den Omega6/Omega3-Quotient sowie Coenzym Q10 und Carnitin.

Ergänzend zu HbA1c und Glukose im nüchternen Zustand bestimmen wir auch den Homa-Index, einen frühzeitigen Marker für eine beginnende Insulinresistenz. Zusätzlich sehen wir uns das Morgencortisol (8-10h) und ACTH an. Dieses Labor wird ergänzt um eine Vollblutmineralanalyse ( $\mathrm{Na}, \mathrm{K}, \mathrm{Ca}, \mathrm{Mg}, \mathrm{Cu}, \mathrm{Zn}$, Selen) sowie ein Neurostressprofil, das ein Cortisol-Tagesprofil aus dem Speichel und die Werte für Serotonin, Adrenalin, Noradrenalin und Dopamin im Urin beinhaltet.

\section{Herzfrequenzvariabilitätsmessung}

Zur Messung der Regulationsfähigkeit des vegetativen Nervensystems führten wir eine Herzfrequenzvariabilitätsmessung inklusive einer Kontrollmessung nach einer angeleiteten Atemübung durch.

\section{Befunde}

Auffälligkeiten fanden sich bei einem Homocysteinspiegel von 19,5 $\mu \mathrm{mol} / \mathrm{I}$ (Normwert < $10 \mu \mathrm{mol} / \mathrm{l})$, Vitamin $\mathrm{B}_{12}$ sehr niedrig mit $354 \mathrm{ng} / \mathrm{l}$ (Normbereich $200-2000 \mathrm{ng} / \mathrm{l}$ ),
Vitamin D 29,7 ng/ml. Im Neurostressprofil niedriges Serotonin mit 104ug/g (Norm: 140-230 $\mathrm{g} / \mathrm{g}$ ), Noradrenalin $26 \mu \mathrm{g} / \mathrm{g}$ (Norm 32-58 $\mathrm{g} / \mathrm{g})$. Dopamin lag mit $164 \mu \mathrm{g} / \mathrm{g}$ (150-280ug/g) im unteren Bereich, Cortisol war erniedrigt im morgendlichen Speichel mit einem Wert von 2,8 ng/ $\mathrm{ml}$ (Normwert 3-9 ng/ml). Im Mineralstoffprofil fanden sich erniedrigte Spiegel für Zink, Magnesium und Selen intrazellulär.

Die Herzfrequenzvariabilitätsmessung ergab eine deutliche neurovegetative Regulationsstörung.

\section{Therapie}

Herr J. erhielt zum Ausgleich der Mikronährstoffmängel folgende Medikation:

- Medivitan i. m. Fertigspritze $5 \times$ im Abstand von jeweils einer Woche, dazu ergänzend ein Vitamin-BKomplett Präparat (Fa. Loges) $1 \times$ täglich morgens

- Zink 25 mg $1 \times$ täglich

- Selen $100 \mu g 1 \times$ täglich

- Magnesiumcitrat 300 mg $2 \times$ täglich (1 Tbl. davon abends)

- Vitamin D mit K2 (Vitamin D + K2 Liquid, Fa. Formmed) täglich 2 Tropfen

Ergänzend erhielt der Patient ein Rosenwurzpräparat (Rhodiola rosea = Vitango) $2 \times$ täglich morgens und mittags. Rosenwurz wirkt sich beruhigend auf Stresssymptome sowie positiv auf depressive Symptome aus [2-5].

Zur Behandlung der Schlafstörungen substituierten wir L-Tryptophan 500 mg 2 Tbl. ca. 60 min vor dem Schlafengehen, was sofort zu einer deutlich besseren Schlafqualität führte.

Der Patient erhielt die Empfehlung, regelmäßig Entspannungsverfahren (Atemübungen $3 \times$ pro Woche 10 min mithilfe der „Vagusvit-App“) sowie moderates Ausdauertraining zu praktizieren. 


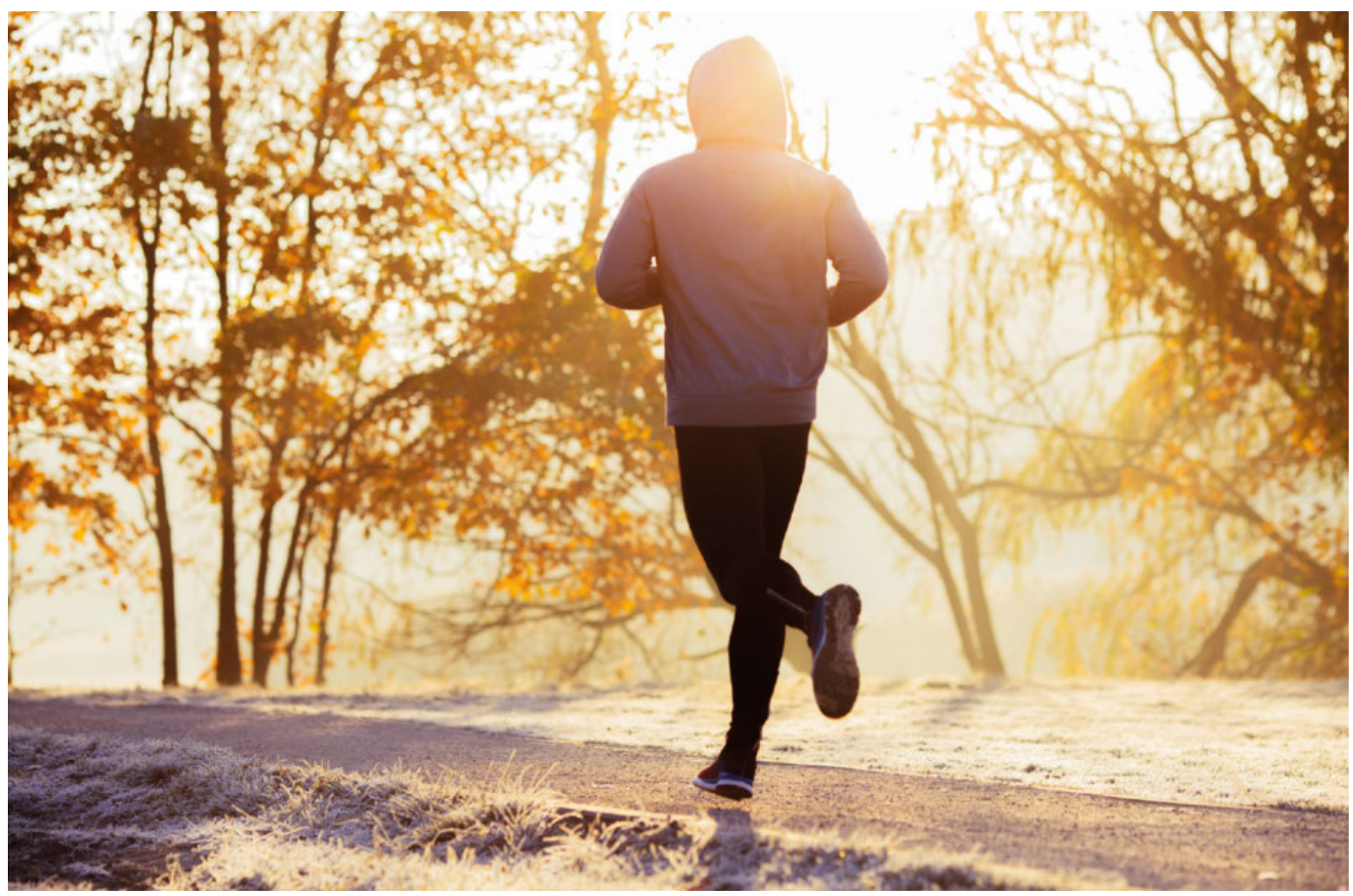

- Abb. 2 Regelmäßige körperliche Bewegung wie Joggen trägt zur körperlichen und mentalen Entspannung bei. @ baranq/Adobe Stock; nachgestellte Situation

Zudem wurde der Patient zur Optimierung der Ernährung beraten: insbesondere zu vollwertigen Kohlenhydraten, hochwertigen Proteinen, guten Fetten insbesondere Omega-3-Fettsäuren, die in Studien Hinweise auf antidepressive Effekte gezeigt haben [6], sowie dem Meiden von Zucker.

Gegen die Kopf -; Nacken - und Schulterschmerzen erhielt Herr J. Akupunkturbehandlungen. Dazu setzten wir Körperakupunktur ein ergänzt um eine vegetativ ausgleichende Ohrakupunktur, wovon der Patient bereits nach der 2. Behandlung profitierte. Insgesamt führten wir 6 Behandlungen durch. Es wurden folgende Punkte genadelt:

Körperakupunktur: Du20, YinTang, He 7, Mi 6, Ni 3, 3E 5, Gb 20, 21, Di 4, Le 3, Gb 34, Ma 36

Ohrakupunktur: Polster, ShenMen, Sonne, Herz, Anti-Aggression, Anti-Frustration

\section{Coaching}

Ergänzend vermittelten wir dem Patienten in ein Coaching. Darin geht es um das Aufdecken der inneren Stressoren und der inneren Antreiber wie „sei perfekt“, „sei gefällig“, „beeil dich“. Diese Verhaltensweisen erlernen wir meist in der frühen Kindheit in unseren Familien oder übernehmen sie von unseren Eltern. Weiter lernt der Patient im Coaching seine Emotionen zu integrieren und von der rein kognitiven Ebene wegzukommen. Viele Menschen neigen dazu, viel zu „verkopft“ an Probleme heranzugehen und dabei die Gefühle vollständig außen vor zu lassen.

Herr J. kann sich mithilfe und unter Anleitung des Coaches in seinen emotionalen Zustand hineinfühlen und es gelingt ihm, seine unerwünschte Erschöpfung zu akzeptieren, mit ihr „Frieden zu schließen“. Hinter einer Erschöpfung finden sich häufig verdrängte Gefühle wie Wut, Trauer oder Verzweiflung. Wird regelmäßig mit den Emotionen gearbeitet, zeigt dieses Vorgehen meist nach kurzer Zeit Erfolge. Herr J. übte täglich und begann auch wieder mit dem Ausdauertraining.

\section{Verlauf}

Diese Behandlungsstrategie mit Mikronährstoffsubstitution, Ernährungsumstellung, Coaching, Achtsamkeitstraining, Wiederaufnahme des Ausdauertrainings sowie der Akupunkturbehandlung führte zu einer raschen Besserung des Befindens. Herr J. berichtete bereits nach kurzer Zeit von einer massiven Besserung seiner Beschwerden, die Tagesmüdigkeit sei vollständig verschwunden. Auch die Ehefrau bestätigt, dass ihr Mann zu Hause nun wieder der alte und von den Aggressionen nichts mehr zu spüren sei.

Die Krankschreibung des Patienten beschränkte sich auf 4 Wochen. Nach dieser kurzen Zeit konnte er in seinen Job zurückkehren, die nächste Beförderungsstufe wieder 
mit Selbstvertrauen erfolgreich angehen, ohne sich erneut über seine Kapazitäten hinaus zu verausgaben.

Derzeit benötigt der Patient keine weitere Behandlung. Wir vereinbarten eine Wiedervorstellung zum Check-up nach einem Jahr, in dem wir präventiv schauen, ob alle Substrate ausreichend vorhanden sind, bzw. frühzeitig Ungleichmäßigkeiten erkennen und ausgleichen können. In unregelmäßigen Abständen hält uns der Patient per E-Mail über sein Befinden auf dem Laufenden.

\section{Fazit}

Der Fall zeigt exemplarisch unsere Erfahrung mit zahlreichen Patienten, dass die Erschöpfung mit einem multimodalen Vorgehen gut behandelt werden kann. Das Vorgehen orientiert sich an den im Vordergrund stehenden Beschwerden des Patienten.

Zunächst nehmen wir die Beschwerden des Patienten sehr ernst. Wir erkennen seine Not und seine Krankheit an. Neben einer ausführlichen Erstanamnese von 60 Minuten, der körperlichen Untersuchung und schulmedizinischen Abklärung wichtiger Differenzialdiagnosen mittels Labor, Ultraschall, Lungenfunktionsprüfung, Durchblutungsmessung und Belastungsergometrie gibt die ergänzende naturheilkundliche Diagnostik häufig die entscheidenden Hinweise. Die Bestimmung des Mikronährstoffstatus mittels Vollblutmineralanalyse, des Neurostressprofils sowie der Herzfrequenzvariabilitätsmessung kann die Standarddiagnostik um wichtige Parameter ergänzt werden.

Das übliche Standardlabor im Patientenbeispiel war vollständig unauffällig. Erst die zusätzlichen Parameter zeigten die behandlungsbedürftigen Mängel an, die zur Erschöpfung des Patienten beigetragen hatten. Das Einbeziehen von Faktoren des Lebensstils wie Ernährung, Bewegung, Stressmanagement, sowie die Aufklärung zu den Zusammenhängen von Lebensführung und Erschöpfung sind Bestandteil des multimodalen Therapieansatzes und haben dem Patienten Instrumente an die Hand gegeben, die er selbst umsetzen konnte und die für den Therapieerfolg sehr wichtig waren.

Gerade bei Erschöpfung ist die Zusammenarbeit von Patient und Therapeut besonders wichtig. Wir können als Ärzte beratend zur Seite stehen, wir können jedoch nicht heilen. Werden für die Erschöpfung zuträgliche Verhaltensweisen fortgesetzt, wird unsere ganzheitliche Therapie nur begrenzt eine Beschwerdebesserung bringen. Gelingt es uns, den Patienten auf einer wertschätzenden Basis zu helfen und zu begleiten, wird nicht nur die Selbstwirksamkeit des Patienten gestärkt, sondern die Therapie auch nachhaltige Erfolge zeigen.
Interessenkonflikt

Die Autorin erklärt, dass keine wirtschaftlichen oder privaten Verbindungen bestehen.

Autorin

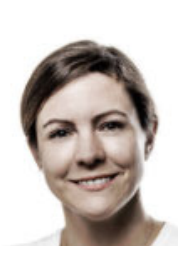

\section{Ruth Biallowons}

Ruth Biallowons ist Fachärztin für Allgemeinmedizin mit ganzheitlichem Schwerpunkt und in der Gemeinschaftspraxis für Ganzheitliche Medizin Düsseldorf niedergelassen. Weiterbildungen im Bereich Naturheilkunde, Akupunktur/Ohrakupunktur, Orthomolekulare Medizin, Natürliche Hormontherapie, Stoffwechselmedizin und Stoffwechseloptimierung. Sie ist Dozentin für die Gesellschaft für Haltungs- und Bewegungsforschung.

\section{Korrespondenzadresse}

\section{Ruth Biallowons}

Gemeinschaftspraxis für Ganzheitliche Medizin Düsseldorf Wildenbruchstr. 115

40545 Düsseldorf - Oberkassel

E-Mail: r.biallowons@googlemail.com

Literatur

[1] Dogs CP, Poelchau N. Gefühle sind keine Krankheit. 5. Aufl. Berlin: Ullstein; 2017

[2] Darbinyan V, Aslanyan G, Amroyan E et al. Clinical trial of Rhodiola rosea L. extract SHR-5 in the treatment of mild to moderate depression. Nord J Psychiatry 2007; 61 (5): 343-348

[3] Hung SK, Perry R, Ernst E. The effectiveness and efficacy of Rhodiola rosea L.: a systematic review of randomized clinical trials. Phytomedicine 2011; 18 (4): 235-244

[4] Olsson EM, von Schéele B, Panossian AG. A randomised, double-blind, placebo-controlled, parallel-group study of the standardised extract shr-5 of the roots of Rhodiola rosea in the treatment of subjects with stress-related fatigue. Planta Med 2009; 75 (2): 105-112

[5] Panossian A, Wikman G, Sarris J. Rosenroot (Rhodiola rosea): traditional use, chemical composition, pharmacology and clinical efficacy. Phytomedicine 2010; 17 (7): 481-493

[6] Grosso G, Pajak A, Marventano S et al. Role of omega-3 fatty acids in the treatment of depressive disorders: a comprehensive meta-analysis of randomized clinical trials. PLoS ONE 2014; 9 (5): e96905

Bibliografie

DOI https://doi.org/10.1055/a-0575-7827

EHK 2018; 67: 104-109

(c) MVS Medizinverlage Stuttgart GmbH \& Co. KG ISSN 0014-0082 